\title{
Pharmacokinetics of Direct Oral Anticoagulants in Emergency Situations: Results of the Prospective Observational RADOA-Registry
}

\author{
Edelgard Lindhoff-Last ${ }^{1,2, *}$ Ingvild Birschmann ${ }^{3, *}$ Joachim Kuhn ${ }^{3}$ Simone Lindau ${ }^{4}$ \\ Stavros Konstantinides ${ }^{50}$ Oliver Grottke ${ }^{6} \quad$ Ulrike Nowak-Göttl $^{7}$ Jessica Lucks ${ }^{2}$ Barbara Zydek ${ }^{2}$ \\ Christian von Heymann $^{8}$ Ariane Sümnig ${ }^{9}$ Jan Beyer-Westendorf ${ }^{10,11,12}$ Sebastian Schellong ${ }^{13}$ \\ Patrick Meybohm ${ }^{4,14}$ Andreas Greinacher ${ }^{9}$ Eva Herrmann ${ }^{15}$ On behalf of the RADOA-Registry \\ Investigators (Reversal Agent use in patients treated with Direct Oral Anticoagulants or vitamin K \\ antagonists Registry)
}

${ }^{1}$ Coagulation Centre at the Cardiology Angiology Centre Bethanien Hospital (CCB), Frankfurt, Germany

${ }^{2}$ Coagulation Research Centre Bethanien Hospital, Frankfurt, Germany

${ }^{3}$ Institute for Laboratory and Transfusion Medicine, Heart and Diabetes Centre, Ruhr University, Bochum, Germany

${ }^{4}$ Department of Anaesthesiology, Intensive Care Medicine and Pain Therapy, University Hospital Frankfurt, Frankfurt, Germany

${ }^{5}$ Center for Thrombosis and Haemostasis (CTH), Johannes Gutenberg University, Mainz, Germany

${ }^{6}$ Department of Anaesthesiology, RWTH Aachen University Hospital, Aachen, Germany

${ }^{7}$ Institute of Clinical Chemistry, Thrombosis \& Haemostasis Treatment Centre, University Hospital, Kiel-Lübeck, Germany

8 Department of Anaesthesia, Intensive Care Medicine, Emergency Medicine and Pain Therapy, Vivantes Klinikum im Friedrichshain, Berlin, Germany

${ }^{9}$ Department of Immunology and Transfusion Medicine,

Universitätsmedizin, Greifswald, Germany
Address for correspondence Edelgard Lindhoff-Last, MD, Cardiology Angiology Center Bethanien Hospital (CCB), Im Prüfling 23, 60389 Frankfurt, Germany

(e-mail: e.lindhoff-last@ccb.de; IBirschmann@hdz-nrw.de).

${ }^{10}$ Thrombosis Research Unit, Department of Medicine 1, Dresden, Germany

11 Division of Haematology, Dresden University Clinic, Dresden, Germany

${ }^{12}$ Department of Haematology and Oncology, Kings College, London, United Kingdom

${ }^{13}$ Medical Department 2, Municipal Hospital, Dresden, Germany

${ }^{14}$ Department of Anaesthesiology, Intensive Care, Emergency and Pain Medicine, University Hospital Wuerzburg, Wuerzburg, Germany

15 Institute of Biostatistics and Mathematical Modelling, Goethe University, Frankfurt, Germany

Thromb Haemost 2022;122:552-559.
Abstract
Keywords
- direct oral anticoagulants
- pharmacokinetics
- emergency
- major bleeding
- urgent surgery

Background Direct oral anticoagulants (DOACs) are increasingly used worldwide. Little is known so far about their pharmacokinetics in emergency situations.

Methods A prospective, observational registry was performed to determine the clinical course in consecutive patients with major bleeding or urgent surgery treated with DOACs. In samples collected as part of routine care DOAC drug concentrations were measured using ultraperformance liquid chromatography-tandem mass spectrometry. Anticoagulant intensity at first presentation and drug half-life $\left(t_{1 / 2}\right)$, tested in repeat samples, were evaluated.

* Both the authors contributed equally to the study.

received

March 19, 2021

accepted after revision

June 25, 2021

published online

July 13, 2021
DOI https://doi.org/ 10.1055/a-1549-6556. ISSN 0340-6245. (c) 2021. The Author(s).

This is an open access article published by Thieme under the terms of the Creative Commons Attribution-NonDerivative-NonCommercial-License, permitting copying and reproduction so long as the original work is given appropriate credit. Contents may not be used for commercial purposes, or adapted, remixed, transformed or built upon. (https://creativecommons.org/ licenses/by-nc-nd/4.0/) Georg Thieme Verlag KG, Rüdigerstraße 14, 70469 Stuttgart, Germany 
Results A total of 140 patients were prospectively included. Pharmacokinetic data were available in $94 \%(132 / 140)$ of patients. Note that $67 \%$ (89/132) experienced lifethreatening bleeding and 33\% (43/132) needed an urgent surgery. For pharmacokinetic analysis a total of 605 blood samples was available. Median concentration on admission was $205 \mathrm{ng} / \mathrm{mL}$ for rivaroxaban and $108 \mathrm{ng} / \mathrm{mL}$ for apixaban. All treatment groups showed a high variation of drug concentrations at baseline. In rivaroxabantreated patients $t_{1 / 2}$ was 17.3 hours ( $95 \%$ confidence interval [Cl]: $15.4-19.7$ ) without significant difference in both groups (major bleeding: $t_{1 / 2} 16.7$ hours, $95 \% \mathrm{Cl}: 14.7-19.3$; urgent surgery: $t_{1 / 2} 19.7$ hours, 95\% Cl: 15.2-27.9; $p=0.292$ ). In apixaban-treated patients $t_{1 / 2}$ was 25.0 hours ( $95 \% \mathrm{Cl}: 22.9-27.6$ ) with a longer $t_{1 / 2}$ after urgent surgery $\left(t_{1 / 2}: 30.8\right.$ hours; $\left.95 \% \mathrm{Cl}: 26.9-36.4\right)$ compared with severe bleeding $\left(t_{1 / 2}: 20.8\right.$ hours; 95\% Cl: 18.8-23.2; $p<0.001)$.

Conclusion Emergency patients under DOAC treatment show a high variation of anticoagulant concentrations at baseline. Compared with rivaroxaban, apixaban showed a lower median concentration on admission and a longer $t_{1 / 2}$.

\section{Introduction}

Patients with nonvalvular atrial fibrillation or venous thromboembolism require therapeutic dose anticoagulation with either vitamin $\mathrm{K}$ antagonists (VKA) or direct oral anticoagulants (DOAC) ${ }^{1-5}$ With the increase in the aging population worldwide, there has been an increase in the prevalence of atrial fibrillation and venous thromboembolism and DOAC have replaced VKA in international guidelines as first choice of anticoagulant for these indications. ${ }^{6,7}$ The risk of intracranial hemorrhage is lower with DOAC compared with VKA, but major bleedings still occur in 1 to $3 \%$ of patients per year. ${ }^{8,9}$ The management of these bleeding complications and of urgent surgery in frail elderly patients under DOAC has become an unmet need in clinical practice and further improvement of care is warranted. ${ }^{9,10}$ However, data on drug levels and pharmacokinetic (pk) of DOAC in these urgent situations are sparse.

Due to the relatively short half-life of DOAC, the timing of the last DOAC dose and the DOAC concentration at the time when the emergency event occurs is important to decide about the use of reversal agents. ${ }^{11-13}$ Peak concentrations of DOAC are reached within 2 to 4 hours after oral intake. The half-lives of these drugs, depending on renal and hepatic function, are usually around 10 to 12 hours. ${ }^{7}$ Drug levels can be determined by drug calibrated anti-factor Xa assays, or the diluted thrombin time (in case of dabigatran), while liquid chromatography-tandem mass spectrometry (LC-MS/MS) is considered the gold standard method for the measurement of DOAC levels. ${ }^{14}$

We have initiated the RADOA-registry (Reversal Agent use in patients treated with Direct Oral Anticoagulants or vitamin $\mathrm{K}$ antagonists), to prospectively assess outcomes in consecutive patients treated with either DOAC or VKA and admitted with major bleeding or with an indication for urgent surgery as recently described..$^{10,15}$ We now report on anticoagulant intensity at first presentation and drug half-life $\left(t_{1 / 2}\right)$ in the DOAC-treated patients of the RADOAregistry. ${ }^{10}$

\section{Methods}

\section{Study Design and Oversight}

The RADOA-registry is a prospective, observational, noninterventional, open-label, investigator-initiated, multicenter clinical registry in Germany documenting the management of severe bleeding and/or urgent interventions in patients under treatment with VKA or DOAC. The rationale and design of the registry have been described previously. ${ }^{10}$

Patients were recruited until the predefined sample size was reached in each group. Patients were then followed prospectively until day 30 after hospital admission.

Participating centers were hospitals with 24-hour interdisciplinary teams to manage anticoagulant-related bleeding in specialized units (i.e., emergency departments and intensive care units). The study protocol was approved by all relevant institutional review boards. An external independent monitor performed $100 \%$ of the onsite source data verification.

\section{Patients}

The inclusion criteria were:

- Age $\geq 18$ years.

- Patients anticoagulated with DOAC or VKA with clinically overt major bleeding according to a modified definition according to the International Society of Thrombosis and Haemostasis for nonsurgical patients ${ }^{16}$ that presented with at least one of the following criteria: symptomatic bleeding in a critical area or organ, such as intracranial, intraspinal, intraocular, retroperitoneal, intra-articular or pericardial, or intramuscular with compartment syndrome or acute life-threatening blood loss leading to hemodynamic instability and/or acute transfusion of two or more units of whole blood or red cells.

- Patients anticoagulated with DOAC or VKA needing an urgent surgical intervention within 24 hours after admission.

The registry started enrolling patients in 2014. The majority of patients was recruited between January 2016 and March 2018. 
Table 1 Baseline characteristics and mortality in patients by treatment and event

\begin{tabular}{|l|l|l|l|}
\hline Variable & Total $(\boldsymbol{n}=132)$ & Major bleeding $(\boldsymbol{n}=89)$ & Urgent surgery $(\boldsymbol{n}=43)$ \\
\hline Male sex, $n(\%)$ & $67(51)$ & $47(53)$ & $20(47)$ \\
\hline Age median (1st-3rd quartile) & $79(72-84)$ & $80(74-84)$ & $77(69-83)$ \\
\hline BMI $\left(\mathrm{kg} / \mathrm{m}^{2}\right)$ median (1st-3rd quartile) & $26(24-30)$ & $26(23-30)$ & $27(24-30)$ \\
\hline Type of bleeding ${ }^{\mathrm{a}}$ & \multicolumn{3}{l|}{} \\
\hline Intracranial/intraspinal, $n(\%)$ & $38(29)$ & $38(43)$ & \\
\hline Gl bleeding, $n(\%)$ & $31(24)$ & $31(35)$ & \\
\hline Other locations, $n(\%)$ & $19(14)$ & $19(21)$ & $23(54)$ \\
\hline Type of surgery ${ }^{\mathrm{a}}(\%)$ & $12(28)$ \\
\hline Trauma, $n(\%)$ & $23(17)$ & & $8(19)$ \\
\hline Acute abdomen, $n(\%)$ & $12(9)$ & & $5(12)$ \\
\hline Other surgery, $n(\%)$ & $12(9)$ & $7(7)$ & \\
\hline Primary endpoint & $12(9)$ & \\
\hline 30-d in-hospital mortality, $n(\%)$ &
\end{tabular}

Abbreviations: BMI, body mass index; GI, gastrointestinal.

${ }^{a}$ Multiple types of bleeding location and surgery are possible.

\section{Ethics}

Due to the emergency nature of the conditions under investigation, patient information and informed consent should not interfere with or delay acute treatment. With the approval of all ethics committees and institutional review boards, written informed consent was obtained from patients after the acute management phase. In the event of a patient's inability to provide written informed consent, this was obtained from his/her legal representative. Data of patients who remained unconscious or died before a legal representative had been appointed were also included. This was explicitly approved by the ethical boards to prevent major bias caused by exclusion of the most severely affected patients. $^{15}$ The study complies with the Declaration of Helsinki.

\section{Substudy of the RADOA-Registry to analyze Pharmacokinetics of DOAC}

In a subgroup of patients included in the RADOA-registry leftover from routine blood samples ("retention blood samples") which were taken during the management of the acute events were collected to analyze drug concentrations and pks of DOAC during these emergency situations ${ }^{10}$ (for additional information concerning recruitment see - Supplementary Fig. S1, available in the online version).

No additional blood sampling for pks was allowed due to the observational character of the registry. Therefore, time points at which these samples were taken were not prespecified and, thus, nonsystematic. Residual citrated plasma samples as well as serum samples were immediately frozen and stored at the participating centers at -20 or $-80^{\circ} \mathrm{C}$ and later shipped on dry ice to the Institute for Laboratory and Transfusion Medicine, Heart and Diabetes Centre, Ruhr University Bochum, Bad Oeynhausen, Germany, where the ultraperformance liquid chromatography (UPLC)-MS/MS analysis of DOAC concentrations was centrally performed.

\section{Sample Preparation and UPLC-MS/MS Analysis of DOACs}

Sample preparation and measurement of dabigatran, rivaroxaban, apixaban, and edoxaban was performed as previously described. ${ }^{17}$ In the majority of cases the measurements were performed in citrated plasma. Predilutions were automatically considered. In case of using serum or lithium heparin samples the concentrations were taken as measured.

In brief, analysis by UPLC-MS/MS was done on a twodimensional (2D) UPLC system (Waters Acquity UPLC H-class with 2D Technology System, Waters GmbH, Eschborn, Germany) directly coupled to a Xevo TQ-S tandem mass spectrometer (Waters $\mathrm{GmbH}$ ) which was operated in electrospray positive ionization mode.

The system control and data acquisition were performed using MassLynx NT 4.1 software with automated data processing by the MassLynx QuanLynx program provided with the instrument. The lower limit of detection for all DOAC of the UPLC-MS/MS method was $<0.2 \mathrm{ng} / \mathrm{mL}$. Since DOAC values $<9 \mathrm{ng} / \mathrm{mL}$ were not clinically relevant, all time points below this value were considered zero. ${ }^{17}$

\section{Statistical Analysis}

The statistical analysis focuses on descriptive statistics (median and range or frequencies where appropriate) and twosided 95\% confidence intervals (CIs). Exponential decay of pks is assessed with linear mixed effect regression models to analyze $t_{1 / 2}$ together with $95 \%$ CIs and to assess associations between the exponential decay and baseline levels. As sensitivity analysis, an analogous but weighted mixed effect regression model was used which gave all patients equal weight to avoid too strong emphasis on patients with a comparable number of observations.

All statistical tests are two-sided and use a significance level of $\alpha=5 \%$ without significance correction for multiple tests. 


\section{Results}

A total of 140 patients treated with DOAC were prospectively included in the RADOA-registry. Residual plasma samples and/or serum samples for measurements of DOAC concentrations were available in 132 patients (94\%) which were further analyzed. The following results only refer to this subgroup of patients.

\section{Patient Characteristics}

For pk analysis a total of 605 blood samples was available. Note that $47 \%(62 / 132)$ of patients were treated with apixaban, 42\% (55/132) with rivaroxaban, 6\% (8/132) with dabigatran, and 5\% (7/132) with edoxaban. One further patient received DOAC treatment (apixaban) as well as VKA treatment (phenprocoumon) because of a medication error and was excluded from the analysis.

Of the evaluable 132 patients, 89 (67\%) experienced a lifethreatening bleeding event and 43 patients (33\%) needed urgent surgery (not driven by severe bleeding) within 24 hours after admission.

Baseline characteristics of patients are given in - Table 1 .

Patients were on average 79 years old. In patients suffering from life-threatening bleeding 43\% (38/89) presented with intracranial or intraspinal hemorrhage and 35\% (31/89) had gastrointestinal bleeding. In patients with urgent surgery, $54 \%(23 / 43)$ had a trauma, mainly fractures and $28 \%$ $(12 / 43)$ needed the intervention because of an acute abdomen. Overall, $9 \%(12 / 132)$ of patients died during the first 30 days after hospital admission.

\section{Rivaroxaban Pharmacokinetics}

In 52 patients treated with rivaroxaban 266 concentration measurements were performed. Time since last intake was available in $64 \%$ of rivaroxaban-treated patients and was 7.2 hours (median; 1 st to 3 rd quartile: $4.4-16.8$ hours). Baseline samples (defined as blood sample taken within 3 hours after presentation) were not available in 13 patients. In the remaining patients rivaroxaban levels showed a high variation from undetectable levels below 9 to $803 \mathrm{ng} / \mathrm{mL}$. A total of 206 rivaroxaban blood samples were available for pk analyses. This analysis included all samples taken during the first 3.5 days after admission ( - Fig. $\mathbf{1}$ ) but excluded patients who were re-exposed to rivaroxaban $(n=3)$. Fitting revealed a mean decay rate of 0.96 per day corresponding to a half-life time of 17.3 hours (95\% CI: 15.4-19.7). There was no significant difference in the decay rate of the baseline levels between patients with severe bleedings and patients requiring urgent surgery (major bleeding: $t_{1 / 2} 16.7$ hours; $95 \% \mathrm{CI}$ : 14.7-19.3; urgent surgery: $t_{1 / 2} 19.7$ hours; $95 \%$ CI $15.2-27.9$, $p=0.292$; - Fig. 1). This result was overall confirmed by means of a sensitivity analysis which resulted in a slightly lower half-life time (for more information see the "Results" section of the additional statistical analysis in the Supplementary Material, available in the online version).

Results of the rivaroxaban levels, creatinine levels, Cockcroft-Gault formula, and coagulation assays during the time course of the registry are shown in $\boldsymbol{-}$ Table 2 . At baseline the

\section{Major bleeding}

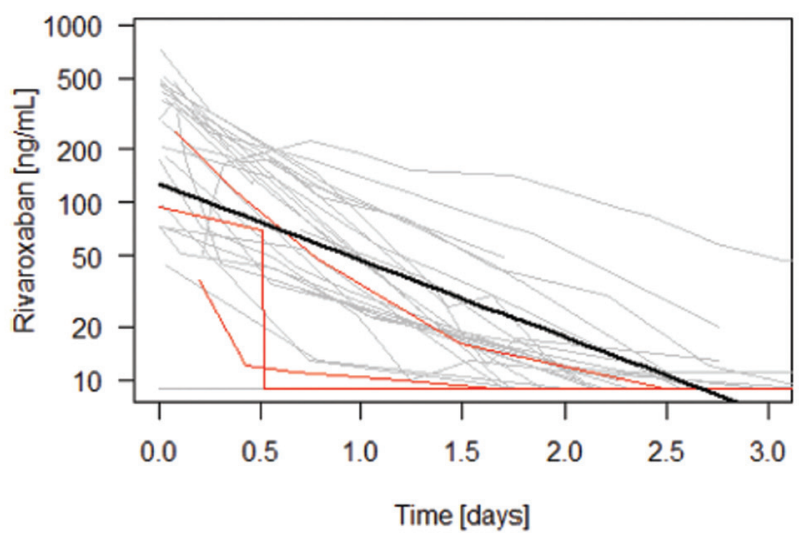

Urgent surgery

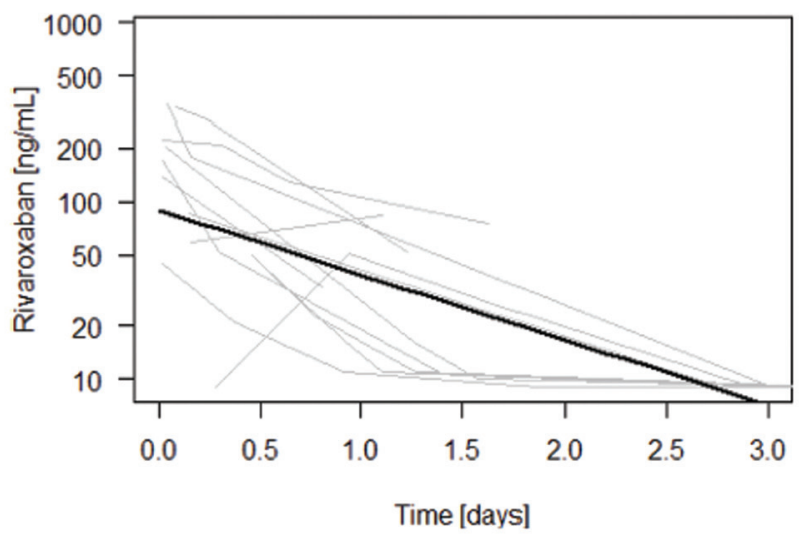

Fig. 1 Results of rivaroxaban concentrations within the first 3.5 days after admission in patients without early re-exposure to rivaroxaban. Patients reaching the 30-day in-hospital mortality are marked by red lines. Thick lines show the line corresponding to the exponential decay from a model that includes the effects and interactions of the treatment groups. Vertical axes are scaled logarithmically. Patients with major bleeding: $n=36,161$ rivaroxaban concentration measurements; Patients with urgent surgery: $n=13,45$ rivaroxaban concentration measurements.

median rivaroxaban level was $205 \mathrm{ng} / \mathrm{mL}, 59 \%$ (23/37) of patients had rivaroxaban levels $>200 \mathrm{ng} / \mathrm{L}$ and $19 \%$ (7/37) presented with rivaroxaban concentrations $\leq 75 \mathrm{ng} / \mathrm{mL}$.

\section{Apixaban Pharmacokinetics}

In 61 patients treated with apixaban 267 concentration measurements were performed. Time since last intake was available in $61 \%$ of the apixaban-treated patients and was 9.9 hours (median: 1 st to 3 rd quartile: $7.1-14.6$ hours). Baseline samples (defined as blood sample taken within 3 hours after presentation) were not available in 15 patients. In the remaining patients apixaban concentrations showed a high variation from undetectable levels below 9 to $1,222 \mathrm{ng} / \mathrm{mL}$.

A total of 221 apixaban blood samples were available for pk analyses. This analysis included all samples taken during the first 3.5 days after admission (-Fig. 2 ) but excluded patients who were re-exposed to apixaban $(n=6)$. 
Table 2 Rivaroxaban levels and laboratory results (number and rates, median, and 1st to 3rd quartile) in 47 patients without reexposure to rivaroxaban within the first week after admission

\begin{tabular}{|l|l|l|l|l|}
\hline Rivaroxaban-treated patients $(\boldsymbol{n}=\mathbf{4 7})$ & Baseline & After $\mathbf{2 4 h}$ & After $\mathbf{~}$-3 d & After 4-6 d \\
\hline Rivaroxaban level $\leq 9 \mathrm{ng} / \mathrm{mL}^{\mathrm{a}}$ & $2 / 37(5 \%)$ & $0 / 29(0 \%)$ & $47 / 79(59 \%)$ & $31 / 40(78 \%)$ \\
\hline Rivaroxaban level $>9$ and $\leq 30 \mathrm{ng} / \mathrm{mL}$ & $0 / 37(0 \%)$ & $16 / 29(55 \%)$ & $20 / 79(25 \%)$ & $5 / 40(13 \%)$ \\
\hline Rivaroxaban level $>30 \mathrm{and} \leq 75 \mathrm{ng} / \mathrm{mL}$ & $5 / 37(14 \%)$ & $8 / 29(28 \%)$ & $8 / 79(10 \%)$ & $2 / 40(5 \%)$ \\
\hline Rivaroxaban level $>75 \mathrm{and} \leq 200 \mathrm{ng} / \mathrm{mL}$ & $9 / 37(23 \%)$ & $5 / 29(17 \%)$ & $4 / 79(5 \%)$ & $2 / 40(5 \%)$ \\
\hline Rivaroxaban level $>200 \mathrm{ng} / \mathrm{mL}$ & $23 / 37(59 \%)$ & $0 / 29(0 \%)$ & $0 / 79(0 \%)$ & $0 / 40(0 \%)$ \\
\hline Rivaroxaban level $(\mathrm{ng} / \mathrm{mL})$ & $205(102-365)$ & $23(16-51)$ & $\leq 9(\leq 9-13)$ & $\leq 9(\leq 9-\leq 9)$ \\
\hline Creatinine $(\mathrm{mg} / \mathrm{dL})$ & $0.9(0.7-1.3)$ & $1.0(0.8-1.6)$ & $0.9(0.7-1.4)$ & $0.8(0.6-1.2)$ \\
\hline Cockcroft-Gault formula $(\mathrm{mL} / \mathrm{min})$ & $66(41-98)$ & $65(44-101)$ & $75(49-133)$ & $63(51-127)$ \\
\hline INR $^{\text {b }}$ & $1.4(1.3-2.0)$ & $1.2(1.1-1.5)$ & $1.1(1.1-1.2)$ & $1.1(1.1-1.2)$ \\
\hline aPTT $(\mathrm{s})^{\text {b }}$ & $32(28-36)$ & $31(28-34)$ & $31(28-35)$ & $32(28-42)$ \\
\hline
\end{tabular}

Abbreviations: aPTT, activated partial thromboplastin time; INR, international normalized ratio; PT, prothrombin time.

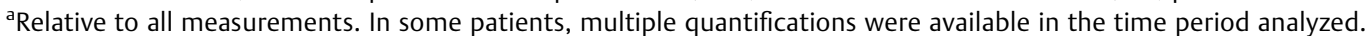

bifferent PT- and aPTT-reagents were used in the participating centers (for more information see - Supplementary Table S1).

Fitting revealed a mean decay rate of 0.66 per day corresponding to a half-life time of 25 hours (95\% CI: 22.9-27.6). There was a significant difference in the decay rate in patients with severe bleedings ( $t_{1 / 2}: 20.8$ hours; $95 \% \mathrm{CI}$ : 18.8-23.2) compared with patients with urgent surgery $\left(t_{1 / 2}: 30.8\right.$ hours; $95 \%$ CI: 26.9-36.4; $p<0.001$; - Fig. 2 ). This result was overall confirmed by means of a sensitivity analysis which resulted in a slightly lower half-life time (for more information see the "Results" section of the additional statistical analysis in the Supplementary Material, available in the online version).

Results of the apixaban levels, creatinine levels, Cockcroft-Gault formula, and coagulation assays during the time course of the registry are shown in - Table 3. On admission the median apixaban level was $108 \mathrm{ng} / \mathrm{mL}, 20 \%$ (9/46) of patients had apixaban levels $>200 \mathrm{ng} / \mathrm{mL}$ and $28 \%(13 / 46)$ presented with apixaban concentrations $\leq 75 \mathrm{ng} / \mathrm{mL}$.

\section{Dabigatran and Edoxaban Pharmacokinetics}

In 8 patients treated with dabigatran 48 concentration measurements were performed. In 7 patients treated with edoxaban 24 blood samples for concentration measurements were available. Time since last DOAC intake was 21.5 hours in edoxaban-treated patients (median: 1st to 3rd quartile: 13.5-28.5 hours) and 7.6 hours in dabigatran-treated patients (median: 1 st to 3rd quartile: $6.4-10.6$ hours). Due to the small sample size statistical analysis of $t^{1} \frac{1}{2}$ was not performed. Results are shown in Appendix B, -Supplementary Figs. $\mathbf{S 2}$ and $\mathbf{5 3}$ (available in the online version).

Table 3 Apixaban levels and laboratory results (number and rates, median, and 1st to 3rd quartile) in 56 patients without reexposure to apixaban within the first week after admission

\begin{tabular}{|l|l|l|l|l|}
\hline Apixaban-treated patients $(\boldsymbol{n}=\mathbf{5 6})$ & Baseline & After $\mathbf{2 4 h}$ & After $2-3 \mathrm{~d}$ & After 4-6 d \\
\hline Apixaban level $\leq 9 \mathrm{ng} / \mathrm{mL}^{\mathrm{a}}$ & $3 / 46(7 \%)$ & $1 / 23(4 \%)$ & $18 / 75(15.0 \%)$ & $24 / 40(60 \%)$ \\
\hline Apixaban level $>9$ and $\leq 30 \mathrm{ng} / \mathrm{mL}$ & $3 / 46(7 \%)$ & $10 / 23(43 \%)$ & $38 / 75(25 \%)$ & $10 / 40(25 \%)$ \\
\hline Apixaban level $>30$ and $\leq 75 \mathrm{ng} / \mathrm{mL}$ & $7 / 46(15 \%)$ & $9 / 23(39 \%)$ & $11 / 75(10 \%)$ & $6 / 40(15 \%)$ \\
\hline Apixaban level $>75 \mathrm{and} \leq 200 \mathrm{ng} / \mathrm{mL}$ & $24 / 46(52 \%)$ & $3 / 23(13 \%)$ & $7 / 75(9 \%)$ & $0 / 40(0 \%)$ \\
\hline Apixaban level $>200 \mathrm{ng} / \mathrm{mL}$ & $9 / 46(20 \%)$ & $0 / 23(0 \%)$ & $1 / 75(1 \%)$ & $0 / 40(0 \%)$ \\
\hline Apixaban level $(\mathrm{ng} / \mathrm{mL})$ & $108(67-181)$ & $32(21-58)$ & $17(10-30)$ & $\leq 9(\leq 9-15)$ \\
\hline Creatinine $(\mathrm{mg} / \mathrm{dL})$ & $1.1(0.8-1.5)$ & $1.0(0.8-1.5)$ & $1.0(0.7-1.6)$ & $0.9(0.7-1.7)$ \\
\hline Cockcroft-Gault formula $(\mathrm{mL} / \mathrm{min})$ & $50(32-79)$ & $60(40-91)$ & $57(32-85)$ & $54(32-76)$ \\
\hline INR $^{\text {b }}$ & $1.3(1.1-1.4)$ & $1.2(1.1-1.5)$ & $1.2(1.1-1.4)$ & $1.1(1.0-1.2)$ \\
\hline aPTT $(\mathrm{s})^{\text {b }}$ & $29(26-34)$ & $30(27-34)$ & $31(28-36)$ & $30(27-40)$ \\
\hline
\end{tabular}

Abbreviations: aPTT, activated partial thromboplastin time; INR, international normalized ratio; PT, prothrombin time.

${ }^{a}$ Relative to all measurements. In some patients, multiple quantifications were available in the time period analyzed.

bifferent PT- and aPTT-reagents were used in the participating centers (for more information see - Supplementary Table S1). 

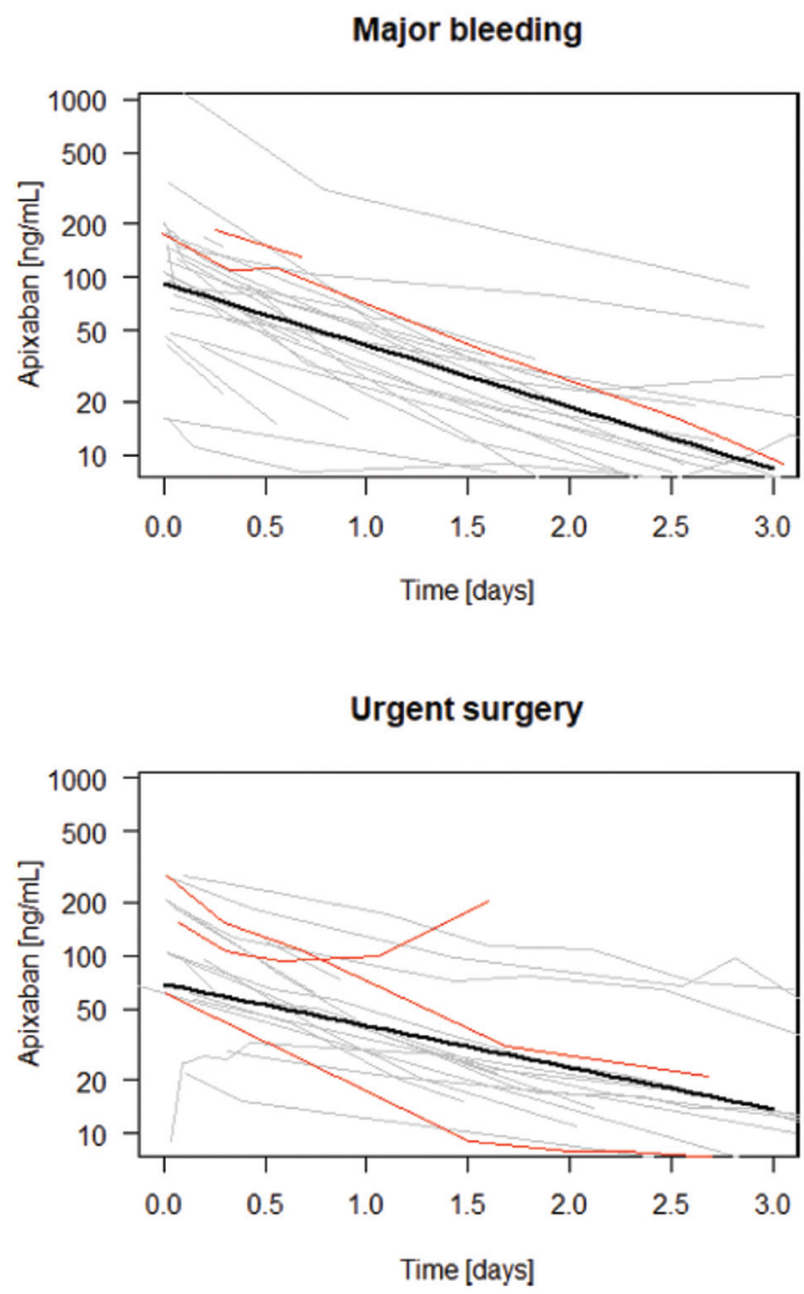

Fig. 2 Results of apixaban concentrations within the first 3.5 days after admission in patients without early re-exposure to apixaban. Patients reaching the 30-day in-hospital mortality are marked by red lines. Thick lines show the line corresponding to the exponential decay from a model that includes the effects and interactions of the treatment groups. Vertical axes are scaled logarithmically. Patients with major bleeding: $n=38,113$ apixaban concentration measurements; Patients with urgent surgery: $n=22,108$ apixaban concentration measurements.

\section{Discussion}

Baseline Anticoagulant Concentrations on Admission In our registry, baseline DOAC concentrations on admission varied widely and ranged from 0 to more than $1,000 \mathrm{ng} / \mathrm{mL}$, which is in agreement with findings from an observational prospective cohort study in which baseline plasma DOAC concentrations were measured in $62 \%$ of 732 DOAC-treated patients with severe bleeding. ${ }^{18}$

In our study baseline median concentrations of rivaroxaban were higher compared with apixaban concentrations due to the once daily intake of a high dose of rivaroxaban compared with apixaban which is applied at lower dosages twice daily.

Median baseline prothrombin time and activated partial thromboplastin time were only slightly prolonged in the DOAC-treated patients. These routine coagulation assays are not sensitive enough to detect clinically relevant residual DOAC concentrations on admission. ${ }^{10}$

\section{Pharmacokinetics of DOAC in Emergency Situations}

To the best of our knowledge this substudy of the RADOAregistry is the first to systematically analyze pks and half-life times in emergency situations under DOAC therapy. The observed $t_{1 / 2}$ of rivaroxaban (17.3 hours) and apixaban (25 hours) in this elderly patient population clearly exceeds the reported $t_{1 / 2}$ of these DOAC in clinical routine (apixaban: 12 hours, rivaroxaban: $11-13$ hours ${ }^{19}$ ) and are in agreement with data of Viktil et al. ${ }^{20}$ In this study evaluating 8 patients with acute hip fractures treated with DOAC, the average elimination half-live was prolonged to 21.6 hours. $^{20}$

Reasons for these significantly prolonged half-lives could be altered pks due to the fragile old patient population and the emergency situation itself. As observed in patients with bleeding events in phase III trials, ${ }^{1-3,5}$ the median age of patients in our registry was 79 years.

At baseline the median Cockcroft -Gault formula was lower in apixaban-treated patients $(50 \mathrm{~mL} / \mathrm{min}$ ) compared with rivaroxaban-treated patients $(66 \mathrm{~mL} / \mathrm{min})$, which might explain the longer $t_{1 / 2}$ of apixaban in our patient population.

Apixaban is cleared via a variety of pathways, including metabolism, biliary excretion, and direct intestinal excretion, with approximately $27 \%$ of total apixaban clearance occurring via renal excretion. Rivaroxaban is eliminated either renally (66\% in total; $36 \%$ unchanged mainly through active renal secretion) or hepatobiliary. ${ }^{21}$ Both drugs have a high degree of plasma protein binding of around $87 \%$ for apixaban and $95 \%$ for rivaroxaban. The volume of distribution is $21 \mathrm{~L}$ for apixaban and $50 \mathrm{~L}$ for rivaroxaban. ${ }^{22,23}$ Redistribution from extravascular compartments may therefore be an additional reason for the prolonged DOAC halflives in our patient population although the patient numbers of our registry are too small to draw definite conclusions.

The results of our study are in contrast to the results of the PAUSE study, which demonstrated that the use of a standardized protocol to stop DOAC treatment before elective procedures resulted in residual DOAC levels below $30 \mathrm{ng} / \mathrm{mL}$ in more than $67 \%$ of patients and less than $5.3 \%$ were above $50 \mathrm{ng} / \mathrm{mL}^{24}$ In contrast, the wide range and high levels of DOAC concentrations observed in our registry are due to the life-threatening clinical situations in which the anticoagulants cannot be stopped in time. In these critical situations rapid and quantitative determination of DOAC concentrations seems to be essential to estimate the bleeding risk in these special patient populations. Thus, all laboratories of hospitals caring for critically ill patients should be able to perform quantitative DOAC measurements in a timely manner, as recommended in the updated International Council for Standardization in Haematology (ICSH) laboratory guidelines for DOAC measurements. ${ }^{25}$

\section{Limitations}

We acknowledge that the RADOA-registry has a nonrandomized observational design. To obtain the highest possible data quality and to minimize any bias, however, patients were 
included prospectively and consecutively, and all enrolled patients were onsite monitored by an independent external monitor. Since we analyzed all residual blood samples available and were allowed to include patients who were unable to provide informed consent, there is minimal selection bias.

\section{Conclusion}

In conclusion, this subgroup analysis of the RADOA-registry shows that baseline concentrations of DOAC differ widely in patients admitted to hospital because of major bleeding or urgent surgery. Quantitative DOAC point of care testing on admission would improve the management of this patient group because only 70 to $80 \%$ of these patients might need specific antidotes on admission due to increased DOAC concentrations. Without rapid DOAC measurements many patients will either receive reversal agents unnecessarily which might increase thrombotic complications in this fragile patient population or may proceed to urgent treatments with high DOAC plasma levels and prolonged anticoagulant activity resulting in prolonged blood loss.

Taken together, these observations suggest the urgent need for quantitative, rapid DOAC measurement availability in all clinical laboratories as recommended by the updated ICSH DOAC laboratory guidance document, ${ }^{25}$ which would be the safer alternative to the just "wait" approach in critically ill patients in these life-threatening situations. Further prospective multicenter studies are necessary to investigate the concentrations of DOACs in the perioperative urgent setting and in major bleeding in higher numbers of patients to be able to correlate these drug concentrations to clinical outcomes and to improve patient care.

\section{What is known about this topic?}

- DOAC have replaced VKA in international guidelines as first choice of anticoagulant for nonvalvular atrial fibrillation and venous thromboembolism. The management of bleeding complications and of urgent surgery in patients under DOAC is a challenge.

- So far data on drug levels and pharmacokinetics of DOAC in these urgent situations are sparse.

\section{What does this paper add?}

- Baseline concentrations of DOACs differ widely in patients admitted to hospital because of major bleeding or urgent surgery. Compared with rivaroxaban, apixaban showed a lower median concentration on admission and a longer $t_{1 / 2}$.

\section{Author Contributions}

E.L.-L. was responsible for the conceptualization and the methodology of the RADOA-registry, organized funding acquisition, and wrote the original draft preparation. I.B. performed the mass spectrometry analysis of the DOAClevels and reviewed major parts of the manuscript. J.K. performed the mass spectrometry analysis of the DOAClevels. S.L., S.K., O.G., U.N.-G., B.Z., C.v.H., I.B., A.S., J.B.-W., S. S., P.M., and A.G. recruited patients and supported the writing of the manuscript. J.L. and B.Z. were responsible for the project administration. E.H. performed the statistical analysis and was responsible for the validation and visualization of the results. All the authors have read and agreed to the published version of the manuscript.

\section{Institutional Review Board Statements}

The study was conducted according to the guidelines of the Declaration of Helsinki, and approved by the Institutional Review Boards of all participating hospitals. For informed consent statements see Ethics as part of the Material and Methods section of the manuscript. ClinicalTrials.gov Identifier: NCT01722786 (URL: https://clinicaltrials.gov/ct2/ show/NCT01722786?term=lindhoff-last\&rank=9).

\section{Funding}

This work was funded by Bayer, Bristol-Myers Squibb/Pfizer, DAIICHI Sankyo, and CSL Behring.

\section{Conflict of Interest}

E.L.-L. has received lecture honoraria and advisory fees from Bayer AG, Boehringer Ingelheim, Bristol-Myers Squibb/Pfizer, Daiichi-Sankyo, Portola, CSL Behring, and Aspen and institutional research support from Bayer AG, Bristol-Myers Squibb/Pfizer, Daiichi-Sankyo, and CSLBehring. I.B. has received speaker's honoraria from Bristol-Myers Squibb/Pfizer, Siemens Healthcare, LFB biomedicaments, and CSL Behring and reimbursement for congress travelling and accommodation from Aspen and Bristol-Myers Squibb. She has performed contract research for Siemens Healthcare and is a member of the advisory board of LFB biomedicaments and of the expert groups of CSL Behring GmbH and Siemens Healthcare Diagnostics Products GmbH. S.K. has received lecture honoraria and advisory fees from Bayer AG, Boehringer Ingelheim, MSD, Actelion, and Daiichi-Sankyo; and institutional research support from Bayer AG, Boehringer Ingelheim, MSD, Actelion, and Daiichi-Sankyo. O.G. has received research funding from Bayer Healthcare, Boehringer Ingelheim, Biotest, CSL Behring, Octapharma, Novo Nordisk, Nycomed, and Portola. He has also received honoraria for lectures and consultancy support from Bayer Healthcare, Boehringer Ingelheim, CSL Behring, Octapharma, Sanofi, Shire, Pfizer, and Portola. U.N.-G. has received lecture honoraria and advisory fees from Bayer AG, Boehringer Ingelheim, Bristol-Myers Squibb/Pfizer, Octapharma, and LFB. C.v.H. has received honoraria for lectures and consultancy work potentially related to this topic, as well as travel reimbursements from Bayer $\mathrm{GmbH}$, Biotest GmbH, Pfizer GmbH, Daiichi Sankyo, CSL Behring, NovoNordisk GmbH, and HICC GbR. J. B.-W. has received personal honoraria (lectures, advisory boards) and travel support from Bayer, Daiichi Sankyo, 
Janssen, and Portola and institutional research support from Bayer, Daiichi Sankyo, Janssen, LEO, Pfizer, and Portola. S.S. has received honoraria for lectures from Bayer, Boehringer, Daiichi Sankyo, and Pfizer, grants, and honoraria from BMS. P.M. has received grants from B. Braun Melsungen, CSL Behring, Fresenius Kabi, and Vifor Pharma for the implementation of Frankfurt's Patient Blood Management program and honoraria for scientific lectures from B. Braun Melsungen, Vifor Pharma, Fearing, CSL Behring, and Pharmacosmos. A.G. has received lecture honoraria and advisory fees from Bayer AG, Boehringer Ingelheim, Bristol-Myers Squibb/Pfizer and Daiichi-Sankyo, ASPEN. The other authors report no conflict of interest. The funders had no role in the design of the registry, in the collection, analyses, or interpretation of data, in the writing of the manuscript, or in the decision to publish the results.

\section{Acknowledgments}

The authors wish to thank the following:

Sina Hehn, MD, Department of Medicine II / University Cancer Center Frankfurt, University Hospital Frankfurt, Germany, who provided support in the development of the online Case Report Form of the Registry;

Sebastian Harder, Department of Clinical Pharmacology, University Hospital Frankfurt, Germany, who gave advice on ethical issues;

Hartmut Clausnizer, MD, Institute of Clinical Chemistry, Thrombosis \& Haemostasis Treatment Center, University Hospital, Kiel-Lübeck, Germany; and

Norbert Weiler, MD, Department of Anesthesiology \& Intensive Care, University Hospital Schleswig Holstein, Campus Kiel, Germany, who recruited patients.

\section{References}

1 Patel MR, Mahaffey KW, Garg J, et al; ROCKET AF Investigators. Rivaroxaban versus warfarin in nonvalvular atrial fibrillation. N Engl J Med 2011;365(10):883-891

2 Connolly SJ, Ezekowitz MD, Yusuf S, et al; RE-LY Steering Committee and Investigators. Dabigatran versus warfarin in patients with atrial fibrillation. N Engl J Med 2009;361(12):1139-1151

3 Granger CB, Alexander JH, McMurray JJ, et al; ARISTOTLE Committees and Investigators. Apixaban versus warfarin in patients with atrial fibrillation. N Engl J Med 2011;365(11):981-992

4 Bauersachs R, Berkowitz SD, Brenner B, et al; EINSTEIN Investigators. Oral rivaroxaban for symptomatic venous thromboembolism. N Engl J Med 2010;363(26):2499-2510

5 Giugliano RP, Ruff CT, Braunwald E, et al; ENGAGE AF-TIMI 48 Investigators. Edoxaban versus warfarin in patients with atrial fibrillation. N Engl J Med 2013;369(22):2093-2104

6 Konstantinides SV, Meyer G, Becattini C, et al; ESC Scientific Document Group. 2019ESC Guidelines for the diagnosis and management of acute pulmonary embolism developed in collaboration with the European Respiratory Society (ERS). Eur Heart J 2020;41(04):543-603

7 Steffel J, Verhamme P, Potpara TS, et al; ESC Scientific Document Group. The 2018 European Heart Rhythm Association Practical Guide on the use of non-vitamin K antagonist oral anticoagulants in patients with atrial fibrillation. Eur Heart J 2018;39(16): 1330-1393

8 Eikelboom J, Merli G. Bleeding with direct oral anticoagulants vs warfarin: clinical experience. Am J Med 2016;129(11S):S33-S40

9 Toorop MMA, Lijfering WM, Scheres LJJ. The relationship between DOAC levels and clinical outcomes: the measures tell the tale. J Thromb Haemost 2020;18(12):3163-3168

10 Lindhoff-Last E. Direct oral anticoagulants (DOAC) - management of emergency situations. Hamostaseologie 2017;37(04):257-266

11 Tripodi A. The laboratory and the direct oral anticoagulants. Blood 2013;121(20):4032-4035

12 Godier A, Dincq AS, Martin AC, et al. Predictors of pre-procedural concentrations of direct oral anticoagulants: a prospective multicentre study. Eur Heart J 2017;38(31):2431-2439

13 Seiffge DJ, Kägi G, Michel P, et al; Novel Oral Anticoagulants in Stroke Patients study group. Rivaroxaban plasma levels in acute ischemic stroke and intracerebral hemorrhage. Ann Neurol 2018; 83(03):451-459

14 Gosselin RC, Adcock DM, Bates SM, et al. International Council for Standardization in Haematology (ICSH) recommendations for laboratory measurement of direct oral anticoagulants. Thromb Haemost 2018;118(03):437-450

15 Lindhoff-Last E, Herrmann E, Lindau S, et al. Severe hemorrhage associated with oral anticoagulants. Dtsch Arztebl Int 2020;117 (18):312-319

16 Schulman S, Kearon CSubcommittee on Control of Anticoagulation of the Scientific and Standardization Committee of the International Society on Thrombosis and Haemostasis. Definition of major bleeding in clinical investigations of antihemostatic medicinal products in non-surgical patients. J Thromb Haemost 2005;3(04):692-694

17 Kuhn J, Gripp T, Flieder T, et al. Measurement of apixaban, dabigatran, edoxaban and rivaroxaban in human plasma using automated online solid-phase extraction combined with ultraperformance liquid chromatography-tandem mass spectrometry and its comparison with coagulation assays. Clin Chim Acta 2018; 486:347-356

18 Albaladejo P, Samama CM, Sié P, et al; GIHP-NACO Study Group. Management of severe bleeding in patients treated with direct oral anticoagulants: an observational registry analysis. Anesthesiology 2017;127(01):111-120

19 Gressenberger P. Reversal strategies in patients treated with direct oral anticoagulants. Vasa 2019;48(05):389-392

20 Viktil KK, Lehre I, Ranhoff AH, Molden E. Serum concentrations and elimination rates of direct-acting oral anticoagulants (DOACs) in older hip fracture patients hospitalized for surgery: a pilot study. Drugs Aging 2019;36(01):65-71

21 Wieland E, Shipkova M. Pharmacokinetic and pharmacodynamic drug monitoring of direct-acting oral anticoagulants: where do we stand? Ther Drug Monit 2019;41(02):180-191

22 Harder S. Pharmacokinetic and pharmacodynamic evaluation of rivaroxaban: considerations for the treatment of venous thromboembolism. Thromb J 2014;12:22

23 Kubisz P, Stanciakova L, Dobrotova M, Samos M, Mokan M, Stasko J. Apixaban - metabolism, pharmacologic properties and drug interactions. Curr Drug Metab 2017;18(07):609-621

24 Tafur AJ, Clark NP, Spyropoulos AC, et al. Predictors of bleeding in the perioperative anticoagulant use for surgery evaluation study.J Am Heart Assoc 2020;9(19):e017316

25 Douxfils J, Adcock DM, Bates SM, et al. 2021 update of the International Council for Standardization in Haematology recommendations for laboratory measurement of direct oral anticoagulants. Thromb Haemost 2021;2021(Mar):19 